\title{
Quantitative Estimation of Structural Change in Carbonyl Iron Powder Compacts during Sintering*
}

\author{
By Ryuzo Watanabe and Yoshimichi Masuda**
}

\begin{abstract}
A quantitative metallographic analysis has been made on the microstructures of carbonyl iron powder compacts sintered in hydrogen at $850^{\circ} \mathrm{C}$. Various parameters for grain, grain boundary and two types of voids, intersected with the grain boundary (void-A) and isolated in the interior of grains (void-B), were determined by the lineal and areal analyses made on the metallographic plane sections. Increase in grain size and decrease in grain boundary area as well as decrease in the number, volume fraction and the area of the free surface of void- $\mathbf{A}$ and the total area of free surfaces could be approximated by the simple power function of sintering time. On the other hand, the isothermal curves for the number, volume fraction and free surface of void-B had a maximum value at a certain period of sintering time. Spheroidization of the voids and the topological change in the sintering structure are also discussed.
\end{abstract}

(Received September 13, 1971)

\section{Introduction}

In the last two decades many studies have been done on the sintering kinetics of monophase metal powders. In most of the studies, changes in shrinkage rate, tensile strength and electrical conductivity during sintering have been measured as a function of time and temperature, and the results have been analyzed on the basis of reaction kinetics and mass transfer theory. These properties of sintered metals are closely related to the features of structures which are characterized by a geometrical configulation of voids and grains. The quantitative estimation of sintering structure is indispensable for the determination of sintering mechanisms since mass flow during sintering should depend upon the change in the structural parameters concerned with the grain and void space. A number of studies have been reported on the grain growth(1), change in void distribution $^{(2)(3)}$, the continuity of void space. and the decrease in inner free surface ${ }^{(4)}$. The last two properties were studied mainly by the school of Rhines on the basis of topology. But these results obtained up to this time seem to be insufficient to describe the sintering process in detail and analyze the sintering mechanisms.

It is the main purpose of this paper to make a quantitative estimation of the change in the microstructure of sintered metal. For the experiment a fine carbonyl iron powder was selected as a model material, since a considerable change in the structure due to the small particle size can be expected during sintering.

\section{Experimental}

The carbonyl iron powder used for this experiment was of MCP grade produced in Mond Nickel Co., and was

* This paper was published originally in Japanese in J. Japan Inst. Metals, 35 (1971), 251.

** Department of Metal Processing, Faculty of Engineering, Tohoku University, Sendai, Japan.

(1) F. Thümmler and W. Thomma : Metals Materials, 1 (1967), 69, (Review 115, p. 84)

(2) F.N. Rhines, C.E. Birchenall and L.A.Hughes : Trans. AIME, 188 (1950), 378. spherical in particle shape. The particle size distribution in Fig. I was determined by the measurement of diameters of 500 particles on photographic planes and the average particle size was 4.5 micron. The powder

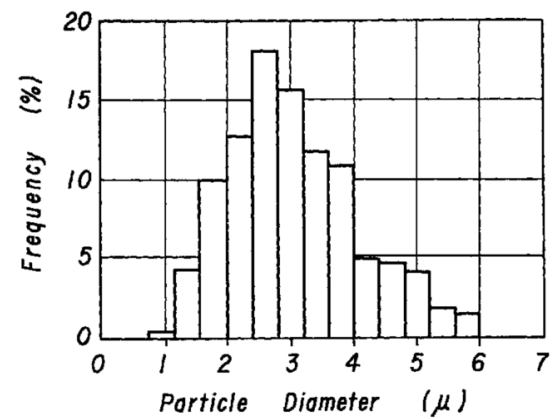

Fig. 1 Size distribution of carbonyl iron powder used in this experiment. Average particle diameter is $3.1 \mu$.

was reduced in purified hydrogen with the dew point of $-70^{\circ} \mathrm{C}$ for an hour at $350^{\circ} \mathrm{C}$ prior to pressing. Then it was compacted at a pressure of 2 tons $/ \mathrm{cm}^{2}$ to a dimension of about $4 \mathrm{~mm}$ wide, $5 \mathrm{~mm}$ in height and 10 $\mathrm{mm}$ long. The compacts were sintered in dried hydrogen at $850^{\circ} \mathrm{C}$ for various periods of time and the temperature was controlled within $\pm 1^{\circ} \mathrm{C}$. The metallographic plane section of the sintered specimens were finished by vibratory polishing with 0.3 and 0.05 micron alumina pastes in order to prevent the deformation of voids and etched with a $2 \%$ nital reagent. Quantitative microscopic measurements were made on the metallographic plane sections with respect to grains, grain boundary and voids as follows. On the photographic planes the numbers of grains, grain boundaries and voids intersected with the unit length of test secant, and the numbers of grains and voids in unit area of the test section were counted. The mean intercept length of grains and voids as well as the lineal fraction of voids

(3) H.F. Fischmeister : International Symposium on Powder Metallurgy, Paris, 1964, Ed. Metaux (St Germain en Laye (1966), p. 155.

(4) F.N. Rhines, R.T. DeHoff and J. Kronsbein : U.S. Atomic Energy Comission Under Contract No. AT-(40-1)-2581. 
were determined by microscopic line scanning. Structural properties determined in this experiment and their symbols, units and the methods of measurements and calculations $^{(5)}$ are listed in Table 1 . In the measurements the voids intersected by grain boundaries were distinguished from those isolated within grains since the mechanisms of shrinkage might be different in the two types of voids. Results of the lineal and areal measurements were applied to the equations listed in Table 1 assuming that the shapes of grains and voids were spherical.

Table 1 Structural properties determined.

\begin{tabular}{l|c|c|c}
\hline \multicolumn{1}{c|}{ Property } & Symbol & Unit & Equation \\
\hline $\begin{array}{l}\text { Volume fraction of } \\
\text { void }\end{array}$ & $f_{v}$ & $(\%)$ & $\begin{array}{c}\text { Lineal fraction } \\
f_{v}=\pi d^{3} N_{v} / 6^{* *}\end{array}$ \\
\hline $\begin{array}{l}\text { Area of } \\
\text { grain boundary and } \\
\text { inner free surface }\end{array}$ & $\begin{array}{c}S_{g} \\
S_{f}\end{array}$ & $\mathrm{~mm}^{2} / \mathrm{mm}^{3}$ & $S=2 N_{L}$ \\
\hline $\begin{array}{l}\text { Grain diameter } \\
\text { Void diameter }\end{array}$ & $\begin{array}{c}d_{g} \\
d_{v}\end{array}$ & $\mu$ & $\begin{array}{l}d=3 \bar{l} / 2^{*} \\
d=4 n / \pi N_{A}^{* *}\end{array}$ \\
\hline Number of voids & $N_{v}$ & $\mathrm{~mm}^{-3}$ & $N_{v}=\pi N_{A}^{2} / 4 n^{* *}$ \\
\hline \hline
\end{tabular}

$N_{L}$ : Number of intersections of grain boundaries and of void surface per unit length of test secant $\left(\mathrm{mm}^{-1}\right)$

$n:$ Number of grains and of voids per unit length of test secant $\left(\mathrm{mm}^{-1}\right)$

$N_{A}$ : Number of grains and of voids per unit area of the test plane $\left(\mathrm{mm}^{-2}\right)$

$\bar{l}:$ Mean intercept length of grains and of voids

* Chord length method ** Simple counting method

\section{Results and Discussion}

\section{Results of the lineal and areal measurements}

The change in the microstructures of the specimens during sintering are shown in Photo. 1. Lineal and areal counts made on such metallographic sections are listed in Table 2 where void-A or void-B are the voids intersected with the grain boundaries or those isolated within grains respectively. Total length of the test line and the total area of the test plane are also listed in Table 2. Mean intercept length of grains and voids and the lineal fraction of voids are shown in Table 3. Structural properties calculated from these data are described in detail in the following sections.

\section{Grain growth}

Increases in grain size during sintering are shown in Fig. 2. Grain size determined by the chord length method agreed well with that by the simple counting method. The isotherm of grain growth is apploximated by a straight line on the logarithmic plot and expressed by the following equation :

$$
d=3.96 t^{0.31}
$$

where $d$ is the grain diameter in microns and $t$ is the time in minutes. The time exponent of eq. (1) accords with that of Kingery's equation ${ }^{(6)}$ for the grain growth during sintering. Kingery derived the growth equation expressed as (2) on the following assumptions: the voids are located on the triple points of grain boundaries which are characteristic of the sintering structure of a crystalline oxide, and move along with the grain boundaries.

$$
d^{3} \sim t
$$

But this is not the case in this experiment; except the early stage of sintering, voids are distributed not only on the triple points but also on any places of grain boundaries and in the interior of grains as shown in Photo. 1. On the other hand, a theoretical equation similar to eqs. (1) and (2) was derived by Hillert for the grain growth of metals in which second-phase precipitates coalesce simultaneously ${ }^{(7)}$. This may suggest,

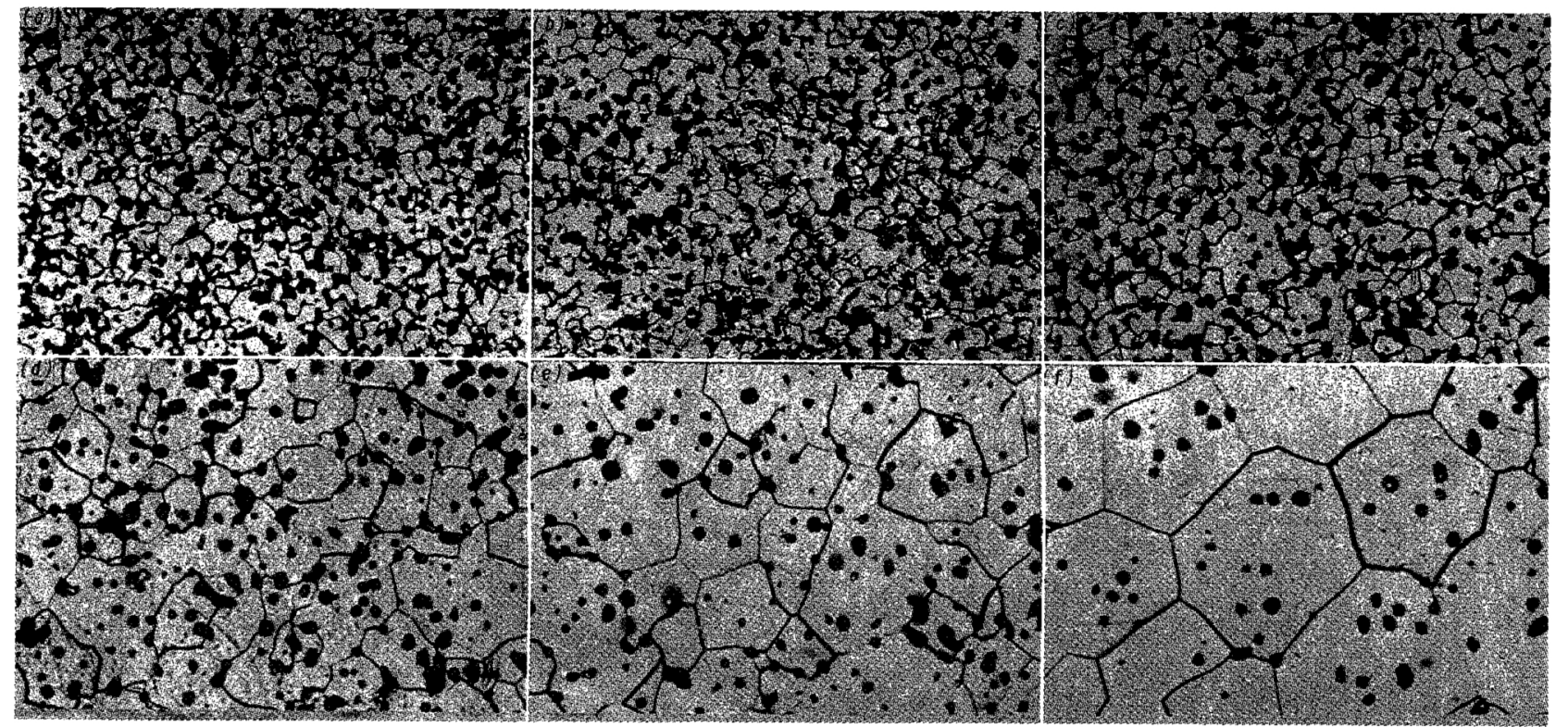

Photo. 1 Change in the microstructure in carbonyl iron powder compacts during sintering in hydrogen at $850^{\circ} \mathrm{C} .(\times 500 \times 3 / 5)$

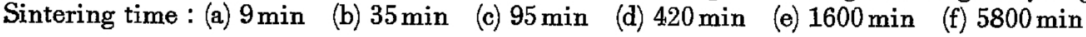

(5) R.T.DeHoff and F.N.Rhines, Eds. : Quantitative Microscopy, McGraw-Hill, (1968).
(6) W.D. Kingery and B. François : J. Amer. Cer. Soc., 48 (1965), 546. 
Table 2 Number of intersections of voids and grain boundaries per unit length of test secant, $N_{L}\left(\mathbf{m m}^{-1}\right)$ and the number of grains and voids per unit area of test plane, $N_{A}\left(\mathrm{~mm}^{-2}\right)$ for carbonyl iron powder compacts sintered in hydrogen at $850^{\circ} \mathrm{C}$.

\begin{tabular}{|c|c|c|c|c|c|c|c|c|}
\hline \multirow{2}{*}{ Time (min) } & \multicolumn{2}{|c|}{ Grain } & \multicolumn{2}{|c|}{ Void-A } & \multicolumn{2}{|c|}{ Void-B } & \multirow{2}{*}{$\begin{array}{c}\text { Length of test line } \\
(\mathrm{mm})\end{array}$} & \multirow{2}{*}{$\begin{array}{l}\text { Test area } \\
\left(\mathbf{m m}^{2}\right)\end{array}$} \\
\hline & $N_{L}$ & $N_{A}$ & $N_{L} / 2$ & $N_{A}$ & $N_{L} / 2$ & $N_{A}$ & & \\
\hline $\begin{array}{r}5 \\
9 \\
15 \\
35 \\
60 \\
95 \\
200 \\
420 \\
680 \\
1600 \\
2580 \\
5800\end{array}$ & $\begin{array}{r}177 \\
154 \\
136 \\
123 \\
103 \\
85 \\
61 \\
45 \\
46 \\
41 \\
33 \\
29\end{array}$ & $\begin{array}{r}31290 \\
29050 \\
27200 \\
11590 \\
12500 \\
5793 \\
3774 \\
1979 \\
1660 \\
1435 \\
785 \\
642\end{array}$ & $\begin{array}{c}103 \\
80 \\
90 \\
51 \\
47 \\
35 \\
25 \\
17 \\
12 \\
7.2 \\
11 \\
3.9\end{array}$ & $\begin{array}{c}\text { ב } \\
\overline{1} \\
10360 \\
11400 \\
9688 \\
6383 \\
3729 \\
3130 \\
2520 \\
1490 \\
889\end{array}$ & $\begin{array}{l}1.6 \\
2.0 \\
3.1 \\
5.0 \\
7.0 \\
11 \\
12 \\
18 \\
15 \\
14 \\
14 \\
11\end{array}$ & $\begin{array}{r}962 \\
1107 \\
1362 \\
1910 \\
2150 \\
3891 \\
3587 \\
4562 \\
4270 \\
3760 \\
3590 \\
2510\end{array}$ & $\begin{array}{r}4.6 \\
4.6 \\
4.6 \\
10.0 \\
10.0 \\
10.0 \\
15.0 \\
15.0 \\
20.0 \\
20.0 \\
20.0 \\
25.0\end{array}$ & $\begin{array}{l}0.035 \\
0.035 \\
0.035 \\
0.070 \\
0.070 \\
0.070 \\
0.13 \\
0.13 \\
0.30 \\
0.30 \\
0.30 \\
0.55\end{array}$ \\
\hline
\end{tabular}

Void-A : voids intersected with the grain boundaries, Void-B : voids isolated within grains

Total length of test line and total area of test plane are listed together.

Table 3 Average chord length of grains and of voids, and volume fraction of voids obtained by lineal and areal analyses for the carbonyl iron powder compacts sintered in hydrogen at $850^{\circ} \mathrm{C}$.

\begin{tabular}{r|r|c|c|c|c|c}
\hline \multirow{2}{*}{$\begin{array}{r}\text { Time } \\
\text { (min) }\end{array}$} & \multicolumn{3}{|c|}{ Chord length $(\mu)$} & \multicolumn{2}{c}{ Volume fraction of void (\%) } \\
\cline { 2 - 7 } & Grain & Void-A & Void-B & Void-A & Void-B & $\begin{array}{c}\text { Void- } \\
\text { (A+B) }\end{array}$ \\
\hline 5 & 4.2 & 2.6 & 0.9 & 27.0 & 0.2 & 27.2 \\
9 & 5.3 & 2.9 & 1.2 & 23.0 & 0.4 & 23.4 \\
15 & 5.3 & 3.5 & 1.3 & 32.0 & 0.5 & 32.5 \\
35 & 8.0 & 2.7 & 1.5 & 13.2 & 1.8 & 15.0 \\
60 & 9.3 & 2.9 & 1.4 & 13.6 & 1.5 & 15.1 \\
95 & 11.3 & 2.8 & 2.0 & 10.3 & 3.4 & 13.7 \\
200 & 15.7 & 3.1 & 2.3 & 7.6 & 4.1 & 11.7 \\
420 & 22.1 & 3.2 & 2.3 & 5.3 & 4.1 & 9.4 \\
680 & 22.6 & 3.4 & 2.5 & 3.8 & 4.0 & 7.8 \\
1600 & 24.1 & 3.2 & 2.9 & 4.3 & 4.1 & 8.4 \\
2580 & 30.0 & 3.8 & 2.2 & 2.7 & 3.7 & 6.4 \\
5800 & 35.3 & 3.4 & 3.2 & 1.4 & 3.0 & 4.4 \\
\hline \hline
\end{tabular}

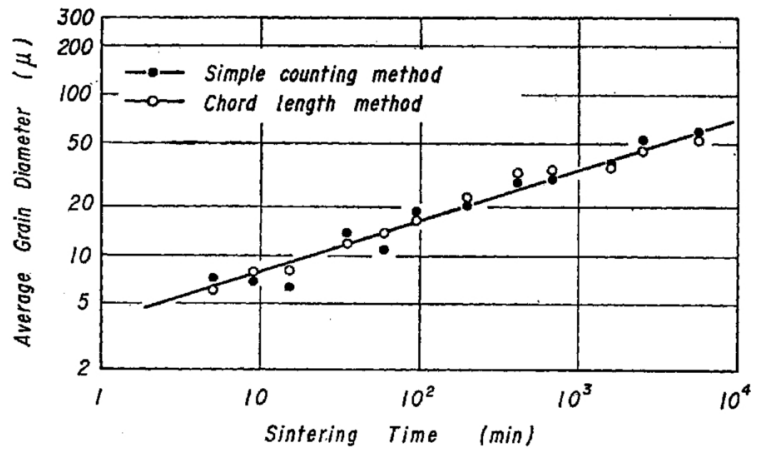

Fig. 2 Isothermal grain growth in carbonyl iron powder compacts during sintering in hydrogen at $850^{\circ} \mathrm{C}$.

together with the characteristic feature of sintering structure, that the voids should be regarded as a kind of inclusions which hinder the grain boundary migration.

\section{Grain boundary area}

As shown in Fig. 3 a linear relationship between grain boundary area and sintering time holds on the logarithmic plot, which is apploximated by the following experimental equation :

$$
S_{g}=5.54 \cdot 10 t^{-0.27}
$$

(7) M. Hillert : Acta Met., 13 (1965), 227.

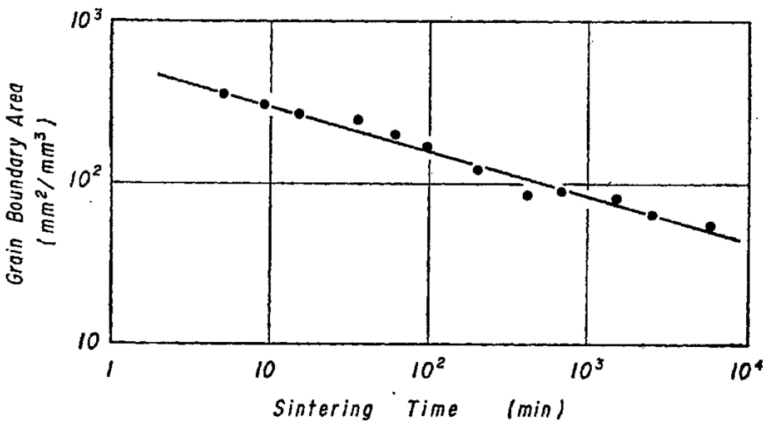

Fig. 3 Logarithms of grain boundary area vs. logarithms of sintering time for carbonyl iron powder compacts during sintering in hydrogen at $850^{\circ} \mathrm{C}$.

where $S_{g}$ is the grain boundary area in unit volume.

The grain boundary acts as a sink of excess vacancies during sintering and it should cause a densification of the compacts ${ }^{(8)}$, so that the shrinkage rate may have to be correlated directly the change in the grain boundary area. However, the change in the grain boundary area is linear on the logarithmic plot, while that in the rate of shrinkage could be divided into the three stages corresponding to the structural changes during sintering ${ }^{(9)}$. This fact may imply that the direction of material flow varies with the change in the relative location of voids and grain boundaries throughout the whole process of sintering.

\section{Porosity}

The changes in the volume fraction of voids during sintering are shown in Fig. 4 and Fig. 5 for the total void, and for the void-A and void-B respectively. The curves for the total void and for the void-A are approximated by the following relations :

$$
\begin{aligned}
& f_{v \text {-total }}=4.43 t^{-0.24} \\
& f_{v-A}=6.71 t^{-0.42}
\end{aligned}
$$

where $f_{v \text {-total }}$ is the total porosity and $f_{v-A}$ is the volume fraction of void-A. It is noteworthy that the volume fraction of void-B has a maximum value at a certain period of sintering time.

(8) W.D. Kingery and M. Berg : J. Appl. Phys., 26 (1955), 1205.

(9) R. Watanabe and Y. Masuda : Unpublished. 


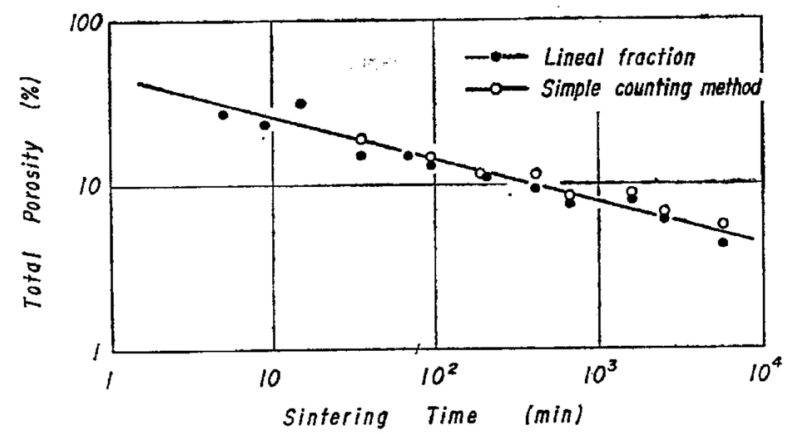

Fig. 4. Relation between total porosity and sintering time.

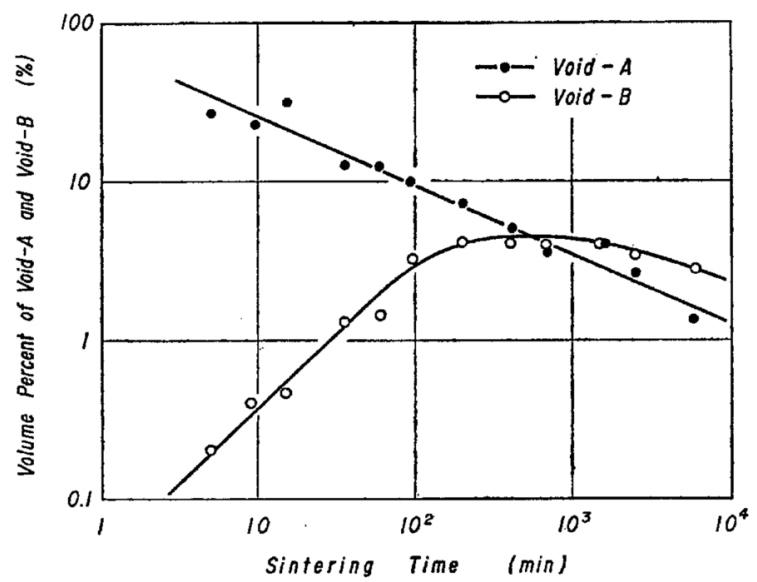

Fig. 5 Logarithms of volume percent of void-A and of void-B vs. logarithms of sintering time for carbonyl iron powder compacts during sintering in hydrogen at $850^{\circ} \mathrm{C}$

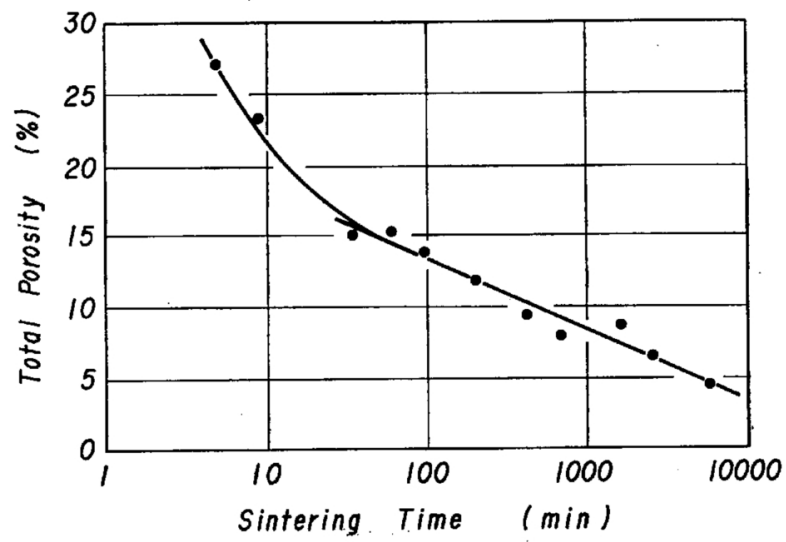

Fig. 6 Total porosity plotted against logarithm of sintering time.

On the relationship between porosity and sintering time Coble ${ }^{(10)}$ derived the following equation for the bulk diffusion mechanism in the intermediate stage of sintering.

$$
p=k \ln t+C
$$

where $p$ is porosity, $k$ and $C$ are constants. Equation (6) can be applied only when the pore space is a continuous multi-channel. The plot of our data deviates remarkably from the logarithmic relation in the early stage of sintering as seen in Fig. 5, whereas the data obtained by Fischmeister ${ }^{(3)}$ for the experimental conditions slightly

(10) R. L. Coble : J. Appl. Phys., 32 (1961), 793. different from ours agreed well with the theoretical equation. It is to be noted, however, that in the later stage our data lies nearly on the straight line and that eq. (6) apparently holds even though the void space becomes discontinuous as represented in the following sections.

\section{Isolation* and spheroidization of voids}

The changes of the void structure during sintering are shown in Photo. 1. In the early stage of sintering the void space forms a continuous multi-channel (Photo. 1 (a)) and is gradually divided into smaller parts with the growth of interparticle necks and the approach of adjacent particle centers (Photo. 1 (b) (c)). The isolation and spheroidization of voids proceeds through the later stage of sintering (Photo. $1(\mathrm{~d}) \sim(\mathrm{f}))$ ). The degree of spheroidization can be estimated by eq. (7) for the lineal and areal measurements ${ }^{(5)}$.

$$
\frac{k_{1} k_{3}}{k_{2}^{2}}=\frac{f_{L} N_{A}}{4 N_{L}^{2}}
$$

where $k_{1}, k_{2}, k_{3}$ are one-, two- and three-dimensional shape factors respectively. The right side term of eq. (7) is determined experimentally and the left side term is a combination of the shape factors. For spherical particles $k_{1}=2, K_{2}=4 \pi, K_{3}=4 \pi / 3$, and $k_{1} k_{2} / k_{2}^{2}=1 / 6 \pi$ $=0.0531$. As the equilibrium shape of voids is spherical, the comparison between the calculated values for the voids of the right side term of eq. (7) and the value for the sphere give a good estimation of spheroidization of voids. The values of eq. (7) calculated from the experimental data listed in Table 3 are given in Table 4. In the case of the voids isolated within grains, the calculated values are near to those of the sphere and no systematic deviation from sphericity can be found. On the other hand, a definite deviation from sphericity can be found in the case of the voids intersected with the grain boundary in the early stage of sintering.

Table 4 Shape parameters of voids in the carbonyl iron powder compacts sintered in hydrogen at $850^{\circ} \mathrm{C}$.

\begin{tabular}{c|c|c}
\hline \hline \multirow{2}{*}{ Time (min) } & \multicolumn{2}{|c}{ Shape parameter } \\
\cline { 2 - 3 } & Void-A & Void-B \\
\hline 5 & $*$ & 0.049 \\
9 & $*$ & 0.069 \\
15 & $*$ & 0.043 \\
15 & 0.033 & 0.062 \\
60 & 0.039 & 0.040 \\
95 & 0.050 & 0.064 \\
200 & 0.047 & 0.062 \\
420 & 0.046 & 0.045 \\
680 & 0.054 & 0.045 \\
1600 & 0.058 & 0.047 \\
2500 & 0.048 & 0.040 \\
5800 & 0.052 & 0.040 \\
\hline \hline
\end{tabular}

\section{Size and number of voids}

Average diameter of voids increases in general with sintering time as shown in Fig. 7. The average diameter of void-A is larger than that of void-B over the

* Isolated void is topologicaly defined to be the one whose genus is zero, and according to this definition the torusshaped void is not isolated. 


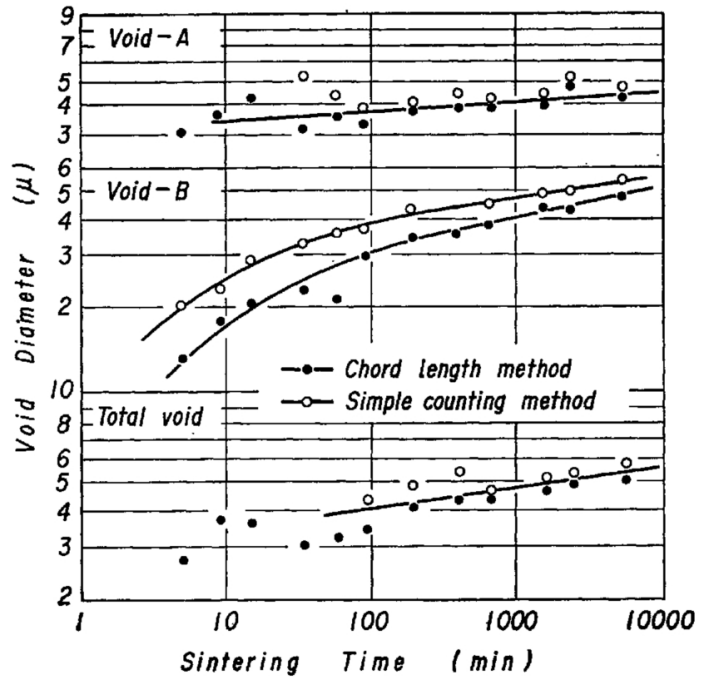

Fig.7 Isothermal growth of voids intersected by grain boundaries (void-A), isolated within grains (void-B) and the average of them (void-A, void-B) in carbonyl iron powder compacts during sintering in hydrogen at $850^{\circ} \mathrm{C}$.

observed time range and the difference between them decreases with sintering time. In addition, experimental values for the size of void-A obtained by the chord length method agreed well with those by the simple counting method. However, in the case of void-B, a remarkable difference has been found between values of void size determined by the two different methods. The experimental errors arising from the two methods will be discussed elsewhere ${ }^{(5)}$.

In Figs. 8 and 9 the change in the total number of voids and the number of two types of voids per unit volume during sintering are represented respectively. The numbers of the total voids and void-A decrease linearly with sintering time on the logarithmic plot. They are apploximated by eqs. (8) and (9) respectively.

$$
\begin{aligned}
& N_{V-\text { total }}=1.54 t^{-0.36} \\
& N_{V-A}=3.00 t^{-0.60}
\end{aligned}
$$

Where $N_{V \text {-total }}$ is the number of voids as a whole and $N_{V-A}$ is the number of void-A. On the other hand, the isothermal curve for the change in the number of

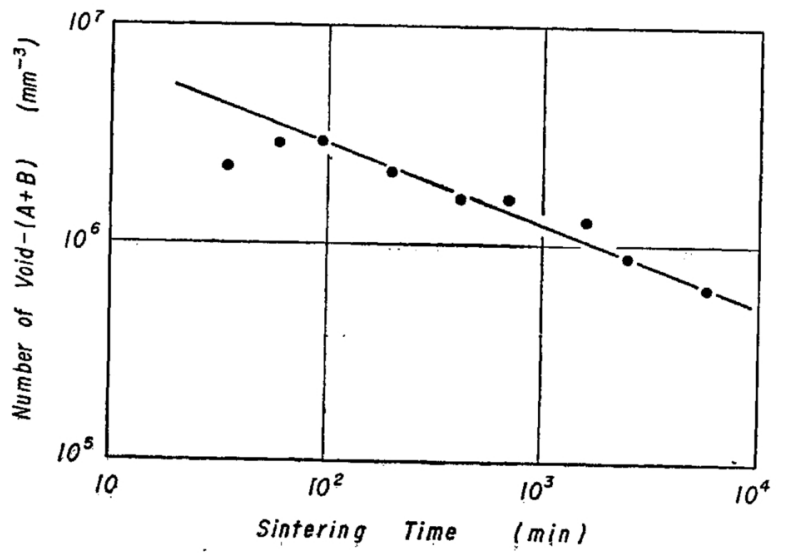

Fig. 8 Relation between totạal number of voids and sintering time.

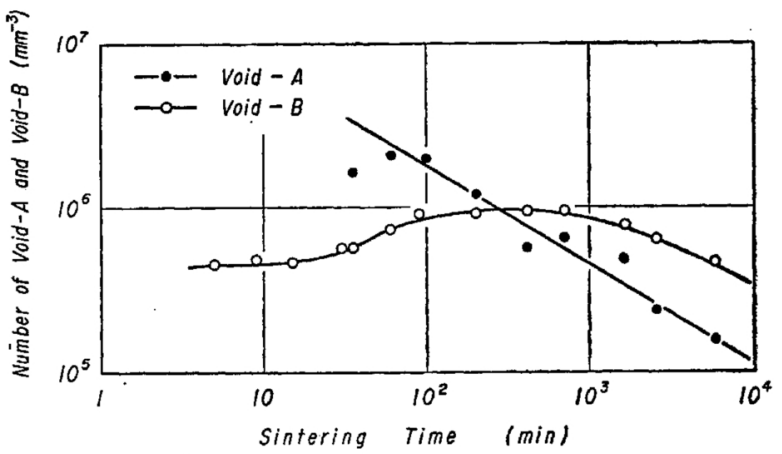

Fig. 9 Logarithms of the number of void-A and of void-B vs. logarithms of sintering time for carbonyl iron powder compacts during sintering in hydrogen at $850^{\circ} \mathrm{C}$.

void-B has a maximum value as in the case of the change in porosity. The number of void-B is of course given by $N_{V-\text { totgl }}$ minus $N_{V-A^{*}}$ The logarithmic plot of the size and number of the void-A deviate from the linearity in the early stage of sintering. This may be caused by the application of a theoretical equation for spherical particles to the voids which were still linked with one another.

\section{Inner free surface}

The inner free surface also changes in a similar way to those of the number and volume fraction of voids. Figure 10 shows the decrease in the total free surface during sintering and the plot is appoximated by the following expression :

$$
S_{f}=6.1 \cdot 10^{2} t^{-0.26}
$$

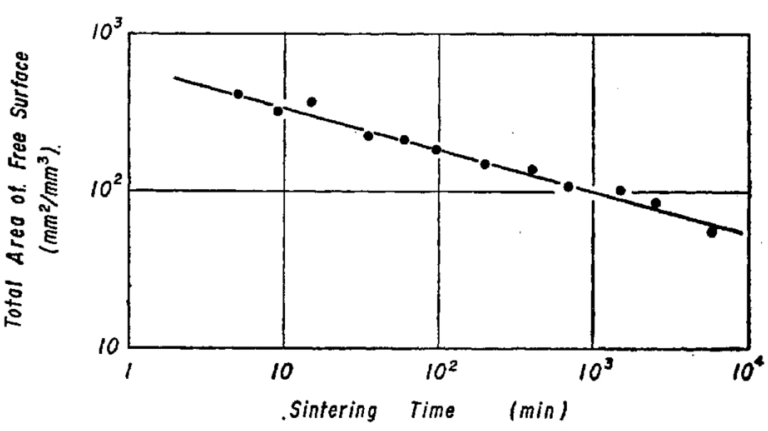

Fig. 10 Relation between total area of free surface and sintering time.

where $S_{f}$ is the total free surface in unit volume. A similar equation to (10) for the change in the total free surface during sintering was also obtained by Fischmeister ${ }^{(3)}$. In Fig. 11 the changes in the free surface of the void-A and the void-B are shown, and the decrease in the free surface of the void-A is approximated by :

$$
S_{f-A}=1.21 \cdot 10^{3} t^{-0.49}
$$

where $S_{f=A}$ is the free surface of the void-A.

Rhines ${ }^{(11)(12)}$ found the following relation between

(11) F. N. Rhines, R. T. DeHoff and R. A. Rummel : Agglomeration, Interscience, New York, 11 (1962), 351.

(12) R. T. DeHoff, R. A. Rummel, H. P. Labuff and F. N. Rhines: Modern Developments in Powder Metallurgy, H. H. Hausner, Ed. Consultants Bureau, New York, 1 (1966), 310. 


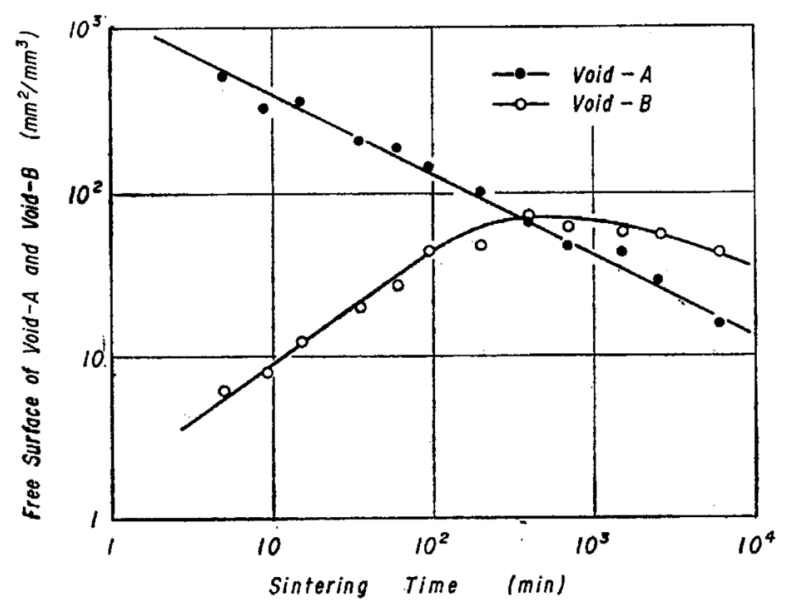

Fig. 11 Logarithms of free surface area of void-A and of void-B vs. logarithms of sintering time for carbonyl iron powder compacts during sintering in hydrogen at $850^{\circ} \mathrm{C}$.

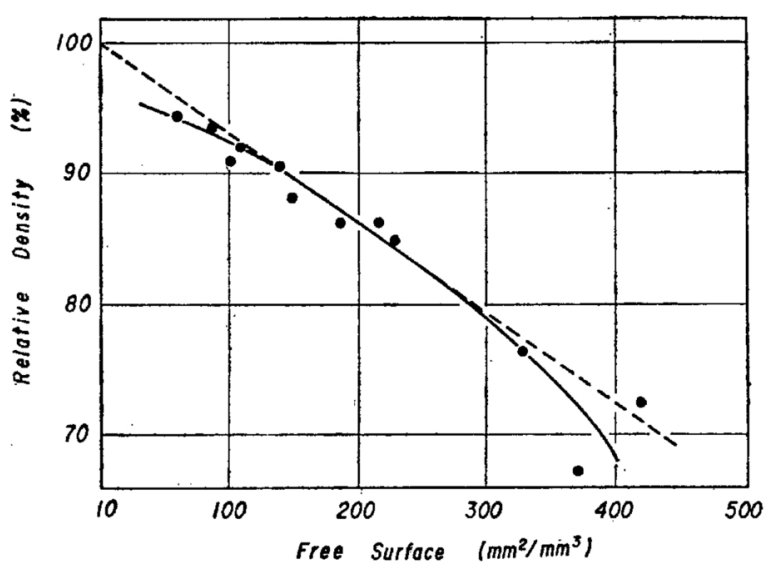

Fig. 12 Relative density plotted against total area of free surface. Broken line represents the relation : $\rho_{a}=m S_{f}+\rho_{t}$

the inner free surface and the apparent density of a sintered body in the intermediate stage of sintering.

$$
\rho_{a}=m S_{f}+\rho_{t}
$$

where $\rho_{a}$ is the apparent density of the compact and $\rho_{t}$ is the true density, $S_{f}$ is the inner free surface and $m$ is a constant. From the theoretical consideration on the basis of topology, $m$ is a function of genus of the three dimensional structure of sintered body ${ }^{(12)}$. The plot of free surface against relative density is shown in Fig. 12. Equation (12) holds only in the density range of about 82 $92 \%$. According to Rhines, the closed packing of the particles cannot be attained in the green compacts and new contacts may be formed by the approach of the centers of the adjacent particles, which causes an increase in the genus of a system. As a result, the deviation from eq. (12) occurred in the early stage of sintering as shown in Fig. 12. The deviation in the high density range is supposed to be caused by the decrease in genus which resulted from the isolation of voids and the decrease in the number of isolated voids. The three ranges in the relation between free surface and density may correspond to the three stages of sintering.

\section{Conclusion}

Structural changes in carbonyl iron powder compacts during sintering at $850^{\circ} \mathrm{C}$ have been studied by quantitative microscopic analysis.

The results are as follows :

(1) The grain size increases according to the onethird power law, in which voids should be regarded to be a kind of inclusions that hinder grain boundary migration.

(2) The grain boundary area decreases according to the simple power function of sintering time.

(3) Average size of voids increases with sintering time. The size of the voids intersected with the grain boundary is always larger than that of the voids isolated within grains over the time range observed and the difference between them decreases with sintering time.

(4) The decreases in total porosity and in total free surface, as well as those in the volume fraction and in the free surface of voids intersected with the grain boundaries, can be approximated by the simple power function of sintering time. On the other hand, the above quantities for the voids isolated within grains have maximum values at a certain period of sintering time.

(5) The change in the total free surface area plotted against relative density is divided into three stages which correspond to the three stages of sintering. 